\title{
The Application of Microscopy in Dairy Science: A Comprehensive Review of Existing and Emerging Techniques
}

\author{
M.A.E. Auty ${ }^{1}$ \\ 1. Teagasc Food Research Centre, Moorepark, Fermoy, Ireland.
}

Microscope innovation has largely been driven by two primary and distinct areas of research: a) biological sciences, where recent developments have focused on maximising resolution of biological materials under dynamic physiological conditions and b) materials science, which tends to be dominated by the physical properties of predominantly hard, relatively solid-state materials. Food ingredients and products do not fit neatly into these categories and require skill and ingenuity in method development as well as a thorough knowledge of food physical chemistry, rheology, and fracture behaviour in addition to biology, biochemistry \& materials science expertise. Interpretation of images derived from food materials also requires experience and there is a need for a holistic approach to characterising food structure that uses the best tools for the job, whether this is basic optical microscopy or the very latest expensive "black box" such as nanoscale X-ray micro-tomography (XMT). In practise, complementary imaging techniques are often necessary to gain a full understanding of the microstructure and a brief review of optical, electron and atomic force microscopy techniques, as applied to industry-relevant dairy ingredients and products will be given. The potential of advanced microscopy techniques such as fluorescence lifetime imaging, confocal Raman microscopy and XMT will also be discussed with examples. This presentation will particularly focus on dynamic techniques that monitor real-time changes in structure at the nano- and micro-length scales during formation and deformation of dairybased food materials and will include time-lapse confocal microscopy, micro-tensile measurement of fracture properties of gels (Fig. 1, [1]) and high speed video-microscopy of powder rehydration. Case studies will be presented to demonstrate how these tools are used in two multidisciplinary projects: a) protein-fat structuring using high dynamic shear processing of milk and its effect on subsequent creaminess perception in low-fat yoghurts, and b) new microscopy techniques to characterise powder hydration. Results indicate that confocal microscopy is particularly useful for characterising protein and fat phases in dairy products and ingredients. Furthermore, a new confocal microscopy technique employing fluorescent tracers to monitor the diffusion of water into single spray dried powder particles is described (Fig. 2). This paper highlights the challenges and opportunities when characterising food materials at the nano- and micro-length scales, particularly given the transient nature of food materials and the complexities of formulation, environmental factors such as temperature, $\mathrm{pH}$ and mechanical processing effects [2].

References:

[1] A.R. Abhyankar et al, Food Hydrocolloids 25 (2011), p275.

[2] This work was supported by the Irish Department of Agriculture, Fisheries and Marine through the FIRM programme [project 15-F-679]. 

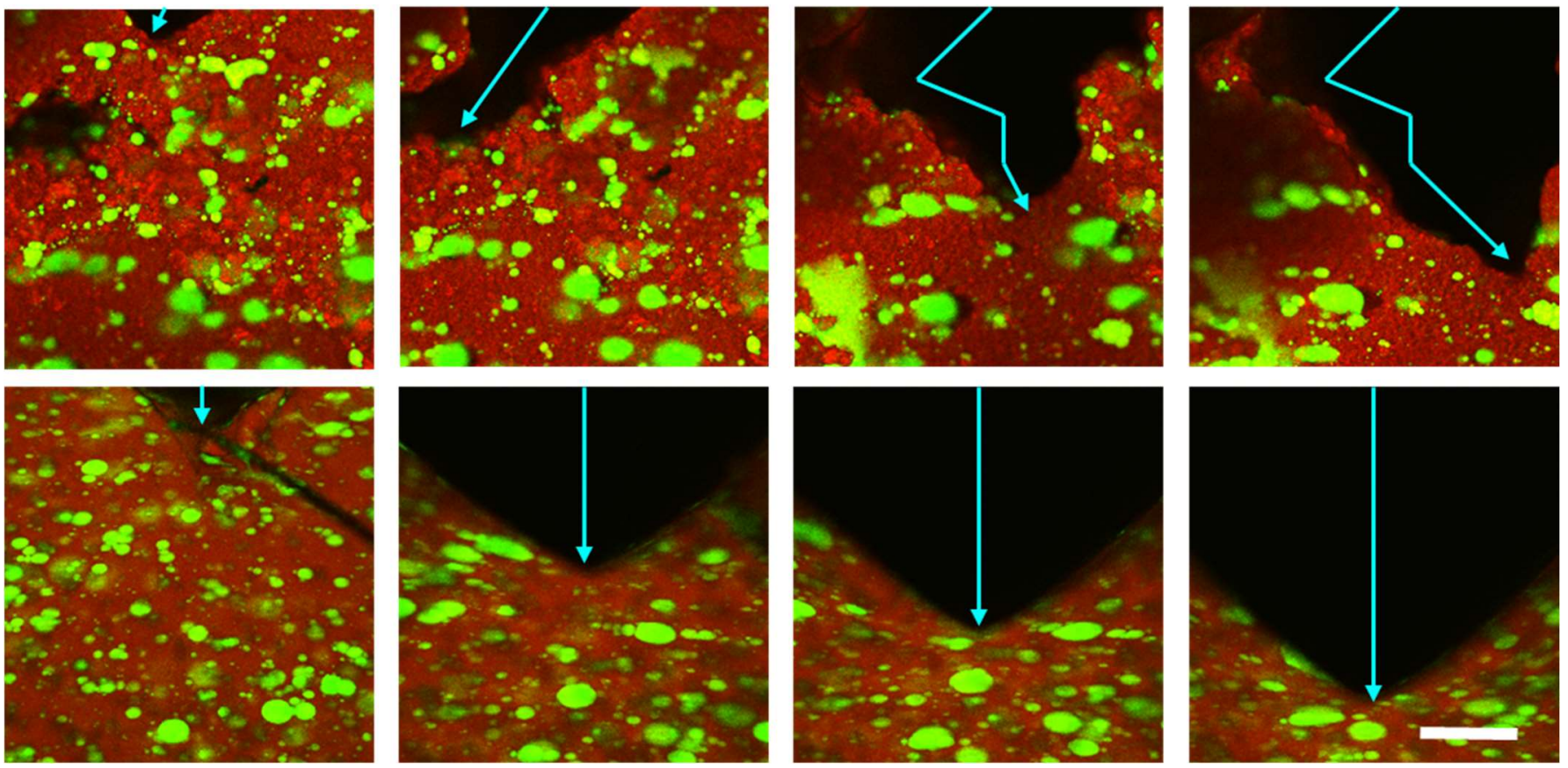

Figure 1. Confocal scanning laser time-lapse micrographs showing fracture behaviour via notch propagation of fat-filled heat-induced whey protein gels (adapted from [1]). Top image series was gelled at $\mathrm{pH}$ 5.6, bottom series gelled at $\mathrm{pH}$ 7.0. Arrows indicate fracture path. Protein matrix is pseudocoloured red, fat droplets are pseudo-coloured green; scale bar is $25 \mu \mathrm{m}$.

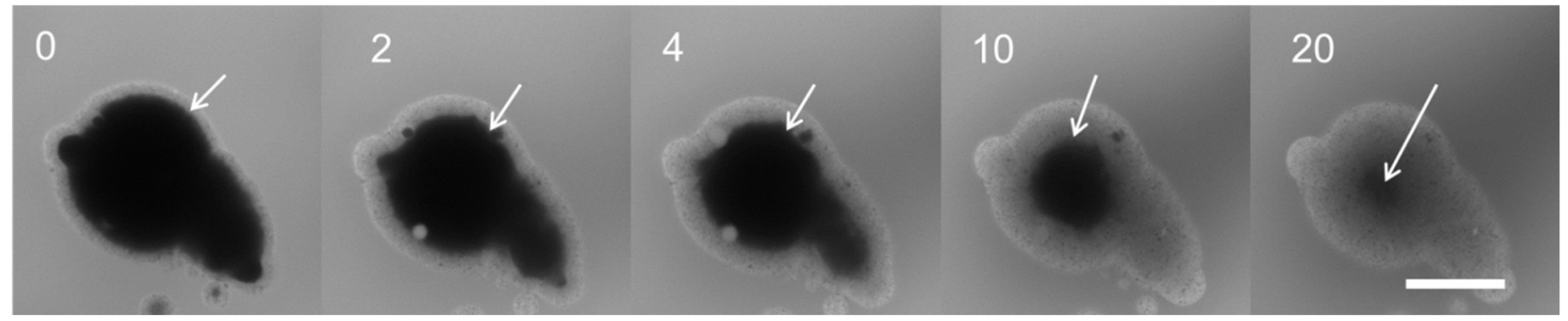

Figure 2. Confocal scanning laser time-lapse micrographs (numbers refer to minutes) of a single spray dried milk particle immersed in an aqueous mixture of water, polyethylene glycol and Rhodamine B. Arrows indicate advancing labelling front; scale bar is $25 \mu \mathrm{m}$. 